\title{
Yüksek Enerjili Rölativistik Çarpışmalarda Baryonik Rezonans Oluşumlari*
}

\section{Production of Baryonic Resonances at High Energy Relativistic Collisions}

\author{
Serpil Yalçın Kuzu1*, Ayben Karasu Uysal ${ }^{2}$ \\ Geliş / Received: 05/01/2021 \\ Revize / Revised: 24/02/2021 \\ Kabul / Accepted: 23/03/2021
}

\begin{abstract}
ÖZ
Baryonik rezonanslar üç kuarktan oluşan uyarılıış durumlardır. Kütle, rezonans genişliği ve ürün bolluğu gibi karakteristik özellikleri ortam tarafından etkilenebilir, böylece bu parçacıkların ölçümleri ile rölativistik ağır iyon çarpışmaları sonucu oluşan sistemin dinamiği araştırılabilir. Çok kısa ortalama ömre $\left(\tau \sim 10^{-23} \mathrm{~s}\right)$ sahip olan baryonik rezonanslar, yüksek enerjili çarpışmalarda oluşan ortamın kimyasal donma noktası ile kinetik donma noktası arasında (i) bozunabilir, (ii) yeniden saçılabilir ve (iii) yeniden üretilebilirler. Bu sebeple bu parçacıkların karakteristik özelliklerinin incelenmesi çarpışmalarda oluşan ve maddenin yeni hali olarak tanımlanan Kuark Gluon Plazma (KGP) ve onu takip eden hadronizasyon safhaları hakkında bilgi verebilir. Ayrıca bu rezonansların farklı yüksek enerjili çarpışma sistemlerinde incelenmesi oluşan ortam boyutlarının rezonans üretimi üzerine etkisini açıklayabilir. Bu çalışmada baryonik rezonanslardan biri ve protonun uyarılmış hali olan $\Delta(1232)^{++}$ rezonansları DPMJET-III olay üreticisi ile $5.02 \mathrm{TeV}$ enerjili proton kurşun $(\mathrm{p}-\mathrm{Pb})$ çarpışmaları için incelenmiştir. Ayrıca elde edilen değerler deneysel sonuçlar ile karşılaştırılmıştır. Farklı rölativistik çarpışma sistemlerinde oluşan ortamın bu parçacık üzerine etkisi değerlendirilmiştir.
\end{abstract}

\section{Anahtar Kelimeler-Baryonik Rezonans, Kütle Kayması, Monte Carlo Olay Üreticileri}

\begin{abstract}
Baryonic resonances, composed of three quarks, are excited states of the corresponding ground state particles. Modification of their properties, such as mass, width, and yield by the medium make them to be used to investigate the dynamics of the system formed in relativistic heavy ion collisions. Due to having very short lifetime $\left(\tau \sim 10^{-}\right.$ ${ }^{23}$ s) these particles may (i) decay, (ii) rescatter, and (iii) regenerate between chemical and kinetic freeze-out temperatures of created medium in high energy collisions. Therefore, studying their characteristic properties provides information about quark gluon plasma (QGP), the new state of matter, and the following phase, hadronization, of the medium formed in collisions. Measurement of these resonances in different high energy collision systems allows explaining system size effect on resonance production mechanism. In this work, $\Delta(1232)$ ${ }^{++}$resonance, which is one of the baryonic resonances and excited state of proton, were studied with DPMJET-III event generator for proton lead $(\mathrm{p}-\mathrm{Pb})$ collisions at the energy of $5.02 \mathrm{TeV}$. In addition, derived values are compared with the experimental results and the effect of the medium formed in different relativistic collisions on the resonances is discussed.
\end{abstract}

Keywords- Baryonic Resonance, Mass Shift, Monte Carlo Event Generators

*Bu çalışma Serpil YALÇIN KUZU'nun doktora tezinden üretilmiștir.

1*Sorumlu yazar iletişim: skuzu@firat.edu.tr (https://orcid.org/0000-0001-8905-8089)

Fizik Bölümü, Fırat Üniversitesi, Fen Fakültesi, Elazı̆̆, Türkiye

2İletişim: ayben.karasu@karatay.edu.tr (https://orcid.org/0000-0001-6297-2532)

Metalurji ve Malzeme Mühendisliği Bölümü, KTO Karatay Üniversitesi, Mühendislik Fakültesi, Karatay, Konya, Türkiye 


\section{GíRiş}

Büyük Patlama (Big Bang) teorisine göre evren bundan 13 milyar yıldan daha uzun bir süre önce çok sıcak ve yoğun tek bir noktadan büyük bir patlama ile oluşmuştur. Patlamayı takip eden birkaç mikro saniye içinde ortam maddenin bilinen en kü̧̈ük yapı taşları olan ve parton olarak da tanımlanan kuark ve gluonların oluşturduğu kuark-gluon plazma (KGP) formuna dönüşmüştür. Zamanın ilerlemesiyle birlikte partonlardan oluşan ortam genişleyerek soğumuş ve serbest durumdaki partonlar, üç kuarklı baryonik ile iki kuarklı mezonik hadronik sistemler haline gelmiştir. Evrenin oluşumunun anlaşılabilmesi için maddenin en küçük yapıtaşlarının serbest olduğu KGP safhası araştııılmalıdır. Bu sebeple laboratuvar koşullarında bu ortamın oluşturulması gerekmektedir.

Rölativistik hadron çarpıştırıcıları, laboratuvar koşullarında çok yüksek sıcaklık ve basınçta güçlü kuvvet yoluyla etkileşen hadronik maddenin özelliklerini çalışmayı sağlayan tek araçtır. Çarpıştırıcıların oluşturduğu şartlar altında hadronik maddenin partonik durum denilen başlangıç durumuna dönmesi beklenir. Avrupa Nükleer Araştırma Merkezi'nde (CERN) Büyük Hadron Çarpıştırıcısı (LHC) $[1,2]$ ve Brookhaven Ulusal Laboratuvarı'nda (BNL) Rölativistik Ağır İyon Çarpıştırıcısı (RHIC) [3] olmak üzere dünyada iki rölativistik hadron çarpıştırıcısı bulunmaktadır. Bu hızlandırıcılarda, çeşitli parçacıklar farklı yüksek enerjilere çıkarılarak çarpıştırılmaktadır. Çarpışmalar sonucu oluşan KGP ortamının özelliklerini inceleyebilmek için büyük dedektör sistemlerine ihtiyaç vardır. Özellikle bu aşamaları araştırmak için LHC'de ALICE (A Large Ion Collider Experiment - Büyük İyon Çarpıştırıcısı Deneyi) [4] ve RHIC'de STAR (Solenoidal Tracker - Solenoid İzleyici) [5] ve PHENIX (Pioneering High Energy Nuclear Interaction eXperiment - Öncü Yüksek Enerjili Nükleer Etkileşim Deneyi) [6] deneyleri tasarlanmıştır.

\section{A. Rölativistik Çarpışmalar}

Rölativistik ağır iyon çarpışmalarında oluşan ortamın dinamiği Şekil 1'de gösterilmiştir [7].Yüksek enerjilerde iki çekirdek çarpıştı̆̆ında başlangıçta çarpışma bölgesinde bulunan nükleonlar etkileşime girerler. $\mathrm{Bu}$ etkileşim sonucu yüksek momentumlu parçacıklar üretilir. Bölgedeki etkileşime girmeyen parçacıklar enerjilerini kaybederek termalleşmeye başlar ve partonların serbest olduğu KGP fazını oluşturur. Bu yoğun ortam genişler ve KGP safhasının hadronizasyon safhasına geçtiği kimyasal donma sıcaklı̆ıına $\left(\mathrm{T}_{\mathrm{c}}\right)$ kadar soğur. Kimyasal donma olarak adlandırılan bu aşamada parçacık türleri sabitlenir, hadronlar arasında esnek olmayan çarpışmalar durur ve ortam parton ve hadron karışımı haline gelir $[8,9,10]$. Artık hadronlardan oluşan bu ortam, parçacıklar arasındaki esnek saçılmaların durduğu kinetik donma sıcaklı̆̆ına $\left(\mathrm{T}_{\mathrm{k}}\right)$ kadar genişlemeye ve soğumaya devam eder $[8,9$, 10].Kinetik donma adı verilen bu aşamada parçacık ürün miktarları sabitlenir. $T_{k}$ 'dan sonra parçacıklar dedektörler tarafindan tespit edilirler.

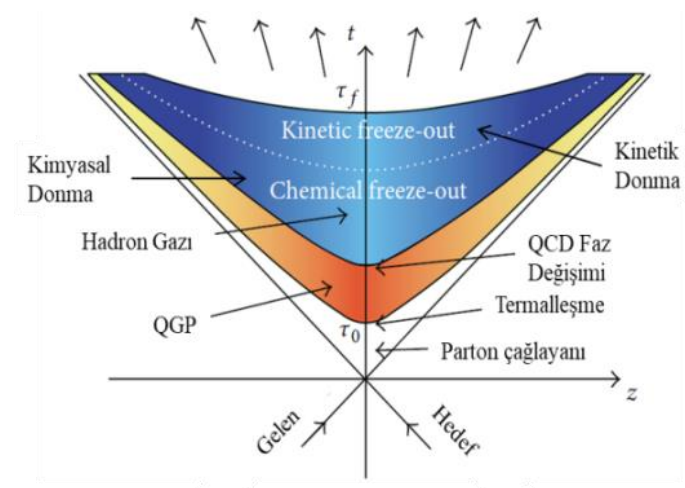

Şekil 1. Yüksek enerjili çekirdek-çekirdek çarpışmalarının uzay(z)-zaman(t) gelişimi [7].

\section{B. Baryonik Rezonanslar}

Baryonik rezonanslar üç kuark yapısına sahip temel durum parçacıklarının uyarılmış halleridir. Bu parçacıklar kararlı emsallerinden farklı spin, yük ve parite gibi kuantum sayılarını taşıyabilirler. Ayrıca karşılık gelen temel durumlarına kıyasla daha yüksek kütle ve geniş̧liğe sahiptirler. Baryonik rezonanslar güçlü kuvvet ile bozunurlar. Bu nedenle yüksek enerjili çarpışmalarda oluşan KGP $[11,12,13,14,15]$ fazının yaşam süresi ile aynı ortalama ömre $\left(\tau \sim 10^{-23} \mathrm{~s}\right)$ sahiptirler [8]. Rezonansları diğer parçacıklardan farklı yapan, bulunduğu ortamın parçacığın özellikleri üzerine etkisidir. Baryonik rezonansların kütle, genişlik ve bolluk gibi özellikleri, içinde 
bulundukları ortam tarafından değiştirilebilir. Bu nedenle, bu parçacıkların oluşumlarının incelenmesi, rölativistik çarpışmalarda oluşan ortamın özellikle kimyasal donma noktası $\left(\mathrm{T}_{\mathrm{c}}\right)$ ile kinetik donma noktası $\left(\mathrm{T}_{\mathrm{k}}\right)$ arasındaki dinamiğini anlamak için önemli bir role sahiptir [9].

Kısa yaşam süreleri nedeniyle baryonik rezonanslar doğrudan ölçülemezler. Fakat dedektörler tarafindan saptanan bozunma ürünlerinin enerjileri $\left(\mathrm{E}_{1}, \mathrm{E}_{2}\right)$ ve momentumlarının $\left(\vec{p}_{1}, \vec{p}_{2}\right)$ Denklem 1'de verilen

$$
m_{\text {inv }}=\sqrt{\left(E_{1}+E_{2}\right)^{2}+\left(\vec{p}_{1}+\vec{p}_{2}\right)^{2}}
$$

değişmez kütle ifadesinde kullanılmasıyla rezonansların değişmez kütle $\left(\mathrm{m}_{\text {inv }}\right)$ dağılımları elde edilebilir. Böylece $\mathrm{m}_{\text {inv }}$ spektrumları ile rezonanslar incelenebilir. Bazı hadronik rezonanslar ve temel özellikleri Tablo 1'de verilmiştir [16].

Tablo 1. Bazı hadronik rezonanslar ve temel özellikleri [16].

\begin{tabular}{lccc}
\hline Rezonans & $\begin{array}{c}\text { Ortalama Bozunma } \\
\text { Uzunluğu c } \tau(\mathbf{f m})\end{array}$ & $\begin{array}{c}\text { Bozunma } \\
\text { Kanalı }\end{array}$ & $\begin{array}{c}\text { Dallanma } \\
\text { Oranı [\%] }\end{array}$ \\
\hline$\rho(770)^{0}$ & 1.3 & $\pi^{+}+\pi^{-}$ & 100 \\
$\Delta(1232)^{++}$ & 1.6 & $\mathrm{p}+\pi^{+}$ & 100 \\
$\mathrm{~K}^{*}(892)^{0}$ & 4.2 & $\mathrm{~K}^{+}+\pi^{-}$ & 66.6 \\
$\Sigma^{*}(1385)^{+}$ & 5.5 & $\Lambda \pi^{+} \rightarrow\left(\mathrm{p} \pi^{-}\right) \pi^{+}$ & 87 \\
$\Lambda^{*}(1520)$ & 12.6 & $\mathrm{p}+\mathrm{K}^{-}$ & 22.5 \\
$\Xi^{*}(1530)^{0}$ & 21.7 & $\Xi^{-} \pi^{+} \rightarrow\left(\Lambda \pi^{-}\right) \pi^{+}$ & 66.7 \\
$\phi(1020)^{0}$ & 44 & $\mathrm{~K}^{+}+\mathrm{K}^{-}$ & 48.9 \\
\hline
\end{tabular}

Baryonik rezonanslar $T_{c}$ ve $T_{k}$ donma noktaları arasında (i) bozunabilir, (ii) yeniden saçılabilir ve (iii) yeniden üretilebilirler. Yeniden saçılma sürecinde rezonansların bozunma ürünleri ve ortamda bulunan hadronlar elastik çarpışma yapabilir. Çarpışmalar parçacıkların momentumlarını değiştirebileceği için bozunma ürünlerinin izleri kaybolabilir. Bu durum rezonans bolluğunda azalmaya sebep olabilir $[17,18]$. Yeniden üretilme sürecinde bozunum ürünleri ile ortamda bulunan hadronlar arasında elastik olmayan etkileşimler meydana gelebilir ve rezonanslar yeniden üretilebilirler. Bu durum rezonans bolluğunda artışa sebep olabilir [17, 18].Şekil 2'de $\Delta(1232)^{++}$baryonik rezonanslarının kimyasal ve kinetik donma sıcaklıkları arasındaki etkileşimleri gösterilmiştir.

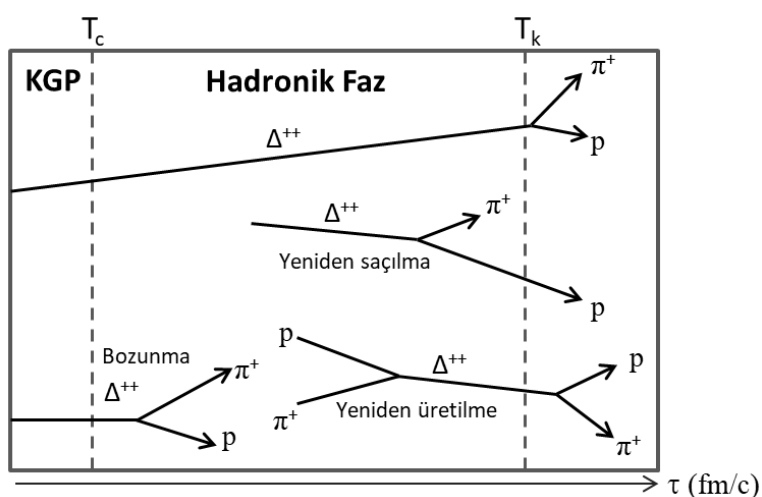

Şekil 2. $\Delta(1232)^{++}$baryonik rezonanslarının kimyasal ve kinetik donma noktaları arasındaki etkileşimleri. Baryonik rezonanslar $\mathrm{T}_{\mathrm{c}}$ ve $\mathrm{T}_{\mathrm{k}}$ donma noktaları arasında (i) bozunabilir, (ii) yeniden saçılabilir ve (iii) yeniden üretilebilirler.

\section{II. $\Delta(1232)^{++}$ÖLÇÜMLERİ}

$\Delta(1232)^{++}$baryonik rezonansı üç yukarı kuarktan (uuu) oluşan protonun uyarılmış bir durumudur. Bu parçacı̆̆ın durgun kütlesi $1.232 \mathrm{GeV} / \mathrm{c}^{2}$ olup yarı ömrü $5.63 \pm 0.14 \times 10^{-24}$ saniyedir [19]. Kısa yarı ömrü nedeniyle $\Delta(1232)^{++}$, diğer hadronik rezonanslar gibi çarpışmalarda oluşan ortamın kimyasal donma noktası ile termal donma noktası arasında çeşitli etkileşmeler yapar ve rezonansın kütle ve genişlik gibi özellikleri yüksek yoğunluk ve sıcaklıktan etkilenir. Bu sebeple $\Delta(1232)^{++}$oluşumlarının incelenmesi ile rezonansın bulunduğu ortam hakkında bilgi edinilebilir. 


\section{A. $\Delta(1232)^{++}$Rezonans Oluşumlarının Deneysel İncelenmesi}

$\Delta(1232)^{++}$ve anti parçacı̆̆ı $\bar{\Delta}(1232)^{--a s ̧ a g ̆ ı d a ~ l i s t e l e n m i s ̧ ~ o l a n ~ h a d r o n i k ~ b o z u n m a ~ k a n a l l a r ı ~ a r a c ı l ı g ̆ ı y l a ~}$ incelenebilirler [16].

$$
\begin{aligned}
\Delta(1232)^{++} & \rightarrow \mathrm{p}+\pi^{+} \\
\bar{\Delta}(1232)^{--} & \rightarrow \overline{\mathrm{p}}+\pi^{-}
\end{aligned}
$$

Dedektörler tarafindan saptanan proton, anti-proton $(\overline{\mathrm{p}})$ ve yüklü piyonların enerji ve momentum bilgilerinin Denklem 1' de verilen değişmez kütle ifadesinde kullanılmasıyla bu parçacıkların belirli bir enine momentum aralığına $\left(\mathrm{p}_{\mathrm{T}}\right)$ ait $\mathrm{m}_{\text {inv }}$ dağılımları elde edilebilir. Elde edilen dağılımlarda rezonansların bozunumları sonucu oluşmayan protonlar ve piyonlar da bulunabilir. Fon (ardalan - background) dağılımı olarak adlandırılan bu parçacıkların dağılımlarını tespit etmek için rezonans analizlerinde uzun zamandır başarıyla kullanılan olaykarıştırma yöntemi (event mixing method - EM) kullanılır [20, 21]. Bu yöntemde $\Delta(1232)^{++}\left(\bar{\Delta}(1232)^{--}\right)$ rezonansının fon spektrumu farklı olaylardan seçilen proton ve piyon çiftlerinin oluşturduğu $\mathrm{m}_{\text {inv }}$ dağılımları kullanılarak belirlenmektedir. EM yönteminde eşleştirilen parçacıkların benzer olaylardan seçilmesi ve aynı parçacıktan gelmemesi bu yöntemin fon dağılımında kullanılmasına olanak sağlar. Fon spektrumundaki belirsizliği düşürebilmek için proton ve piyonlar birden fazla farklı olaydan seçilir ve rezonansın değişmez kütle hesaplaması yapılır. Fon dağılımındaki veri sayısı, oluşturulan $\Delta(1232)^{++}\left(\bar{\Delta}(1232)^{--}\right) \mathrm{m}_{\text {inv }}$ dağılımındaki veri sayısından daha fazladır. Bu nedenle EM sonucu elde edilen spektrumlar parçacığın değişmez kütlesinin beklenmediği bir aralığa göre normalize edilir[18]. Normalizasyon faktörü, rezonansın değişmez kütle sinyalinin beklenmediği bir aralık için aynı olay proton-piyon çiftlerinin oluşturduğu dağılımdaki veri sayısının farklı olaylardan elde edilmiş proton-piyon çiftlerinin oluşturduğu fon dağılımına oran ıalınarak hesaplanır[18]. Delta rezonansının kütlesinin $1.232 \mathrm{GeV} / \mathrm{c}^{2}$ olmasısebebiyle,normalizasyon için kullanılacak kütle aralığı genellikle 1.4$1.8 \mathrm{GeV} / \mathrm{c}^{2}$ alınır. Bir çarpışma olayındaki proton-piyon çiftlerinin değişmez kütle dağılımlarından, normalize edilmiş fon dağılımları çıkartılarak rezonansın $m_{\text {inv }}$ spektrumu elde edilir. Şekil 3'de STAR deneyinde ölçülmüş $200 \mathrm{GeV}$ kütle merkezi enerjili $\left({\sqrt{\mathrm{S}_{\mathrm{NN}}}}\right)$ orta hizlılık (rapidite) $(|\mathrm{y}|<0.5)$ değerlerinde döteron-altın $(\mathrm{d}+\mathrm{Au})$ ve protonproton (pp) çarpışmaları sonucu oluşan $\Delta(1232)^{++}$rezonanslarının değişmez kütle dağılımları $0.6-0.8 \mathrm{GeV} / \mathrm{c}$ enine momentum aralığı için gösterilmiş̧ir[18].

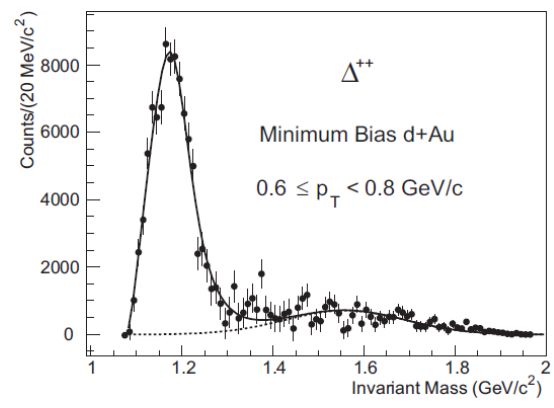

(a)

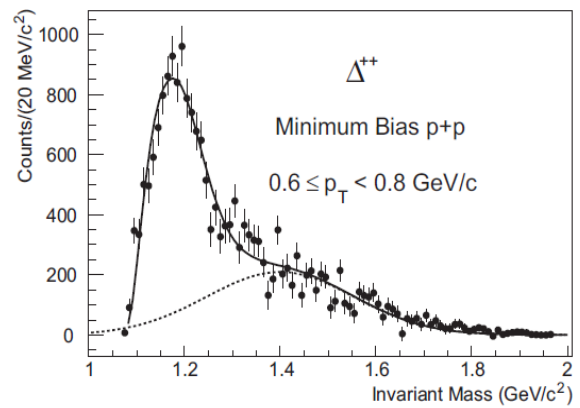

(b)

Şekil 3. STAR deneyinde ölçülmüş $200 \mathrm{GeV}$ kütle merkezi enerjili $\left(\vee_{\mathrm{S}_{\mathrm{NN}}}\right)$ orta hızlılık $(|\mathrm{y}|<0.5)$ değerlerinde döteron-altın $(\mathrm{d}+\mathrm{Au})(\mathrm{a})$ ve proton-proton (pp) (b) çarpışmaları sonucu oluşan $\Delta(1232)^{++}$rezonansının $0.6<\mathrm{p}_{\mathrm{T}}<0.8 \mathrm{GeV} / \mathrm{c}$ enine momentum aralığı için değişmez kütle dağılımları [18]. Grafiklerdeki hata çubukları istatiksel belirsizlikleri göstermektedir. Rezonans dağılımları faz uzay faktörü uygulanmış pdalga Breit - Wigner fonksiyonu ile modellenmiş̧ir ve grafiklerde siyah çizgiler ile gösterilmiş̧ir. Rezonansa ait olmayan proton-piyon çiftleri lineer fonksiyon kullanılarak modellenmiştir ve grafiklerde kesik çizgiler ile gösterilmiş̧ir.

$\Delta(1232)^{++}$rezonans1 izospin değeri $\mathrm{I}=3 / 2$, toplam açısal momentumu $\mathrm{J}=3 / 2$ ve yörünge açısal momentumu $1=1$ olan bir piyon-nükleon durumudur. Bu durumun $\mathrm{P}_{33}(1232)$ kuantum hali ile ifade edilmesi sebebiyle rezonans dağılımları faz uzay faktörü (phase space factor - PSF)uygulanmış p-dalga Breit - Wigner fonksiyonu ile tanımlanır [22, 23, 24, 25]. Şekil 3'de siyah çizgiler $\Delta(1232)^{++}$rezonans dağılımlarının faz uzay faktörü uygulanmış p-dalga Breit - Wigner fonksyonu ile modellenmesini göstermektedir. Şekilde de görüldüğü gibi fon dağılımlarının çıkartılmasına rağmen dağılımlarında rezonansa ait olmayan proton-piyon çiftleri gözlenmektedir. $\mathrm{Bu}$ ardalan çiftlerin dağılımı Gauss fonksiyonu ya da lineer fonksiyon kombinasyonları kullanılarak tanımlanabilir. Şekil 3'de kesik çizgiler bu çiftlerin lineer fonksiyon kullanılarak modellenmesini 
göstermektedir [18]. Faz uzay faktörü uygulanarak elde edilen p-dalga Breit - Wigner fonksiyonu ile parçacı̆̆ın farklı enine momentum aralıkları için kütle ve rezonans genişlikleri elde edilir. Şekil 4'de STAR deneyi ${\sqrt{\mathrm{S}_{\mathrm{NN}}}}=$ $200 \mathrm{GeV}$ enerjili orta hızlılık $(|\mathrm{y}|<0.5)$ değerlerinde $\mathrm{d}+\mathrm{Au}$ ve pp çarpışmaları sonucu oluşan $\Delta(1232)^{++}$rezonanslarının0.2ile1.6 GeV/c p p $_{\text {T }}$ aralığı için değişmez kütle değerleri gösterilmiştir [18]. Şekilde düz çizgiler rezonansın Parçacık Data Grubunda (PDG) [16]tarafından belirtilmiş referans kütle değerini (1.232 $\mathrm{GeV} / \mathrm{c}^{2}$ ) temsil etmektedir. STAR deneyinde yapılan bu iki çalışma rezonansın kütlesinde enine momentuma bağlı bir kayma olduğunu tespit etmişstir.
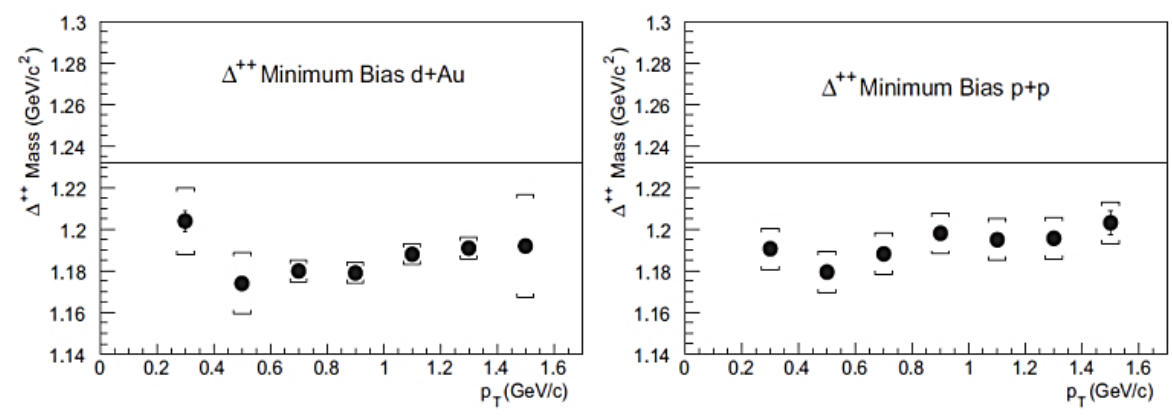

Şekil 4. STAR deneyinde ölçülmüş olan ${\sqrt{S_{N N}}}=200 \mathrm{GeV}$ enerjili orta hızlılık $(|\mathrm{y}|<0.5)$ değerlerinde döteron-altın $(\mathrm{d}+\mathrm{Au})($ sol) ve protonproton (pp) (sağ) çarpışmaları sonucu oluşan $\Delta(1232)^{++}$rezonansının $0.2-1.6 \mathrm{GeV} / \mathrm{c}$ p $\mathrm{T}$ aralığı için kütle değerleri [18]. Grafiklerdeki hata çubukları istatiksel belirsizlikleri parantezler ise sistematik belirsizlikleri göstermektedir. Düz çizgiler rezonansın PDG tarafından yayınlanmış kütle değerini göstermektedir.

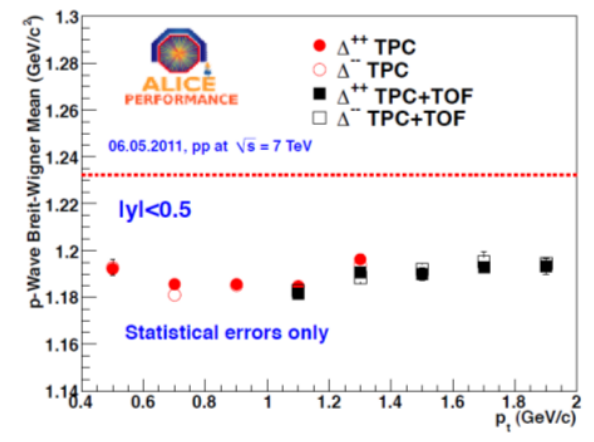

Şekil 5. ALICE deneyinde ölçülmüş olan $V_{\mathrm{S}}=7 \mathrm{TeV}$ orta hızlılık $(|\mathrm{y}|<0.5)$ pp çarpışmaları sonucu oluşan $\Delta(1232)^{++}$ve $\bar{\Delta}(1232)^{--}$ rezonanslarının $0.4<\mathrm{p}_{\mathrm{T}}<2.0 \mathrm{GeV} / \mathrm{c}$ aralığı için kütle değerleri [26]. Grafikteki hata çubukları istatiksel belirsizlikleri temsil etmektedir. Kesikli çizgi rezonansın PDG tarafından kabul edilmiş kütle değerini göstermektedir.

$\Delta(1232)^{++}$kütle ölçümleri daha yüksek enerjiler için ALICE deneyinde çalışılmıştır. Şekil 5'de ALICE deneyi $\sqrt{s}_{\mathrm{s}}=7 \mathrm{TeV}$ enerjili orta hızlılık $(|\mathrm{y}|<0.5)$ değerlerinde pp çarpışmaları sonucu oluşan $\Delta(1232)^{++} \mathrm{ve} \bar{\Delta}(1232)^{-}$ - rezonanslarının $0.4<\mathrm{p}_{\mathrm{T}}<2.0 \mathrm{GeV} / \mathrm{c}$ aralığı için kütle değerleri gösterilmiştir [26]. Şekilde kesikli çizgiler rezonansın PDG[16] tarafından yayınlanmış kütle değerini ifade etmektedir. ALICE deney sonuçları rezonansın kütlesinde enine momentuma bağlı bir kayma olduğunu göstermiştir.

\section{B. Monte Carlo (MC) Simülasyonu ile $\Delta(1232)^{++}$Kütle Değerlerinin Elde Edilmesi}

$\mathrm{Bu}$ araştırmada, $\Delta(1232)^{++}$rezonanslarının yüksek enerjili çarpışma sistemlerinde oluşumları Monte Carlo olay üreticilerinden biri olan DPMJET-III ile çalışılııştır [27]. Bu model Dtunuc-2 [28, 29], Dpmjet-II [30, 31] ve Phojet1.12[32, 33] olay üreticilerinin birleştirilmiş versiyonudur [27]. DPMJET-III, birkaç GeV' den yüksek enerjilere $\left(\sim 10^{11} \mathrm{GeV} /\right.$ nükleon$)$ kadar hadron-hadron, hadron-çekirdek, çekirdek-çekirdek, foton-hadron, fotonfoton ve foton-çekirdek etkileşimlerinin simülasyonunu sağlar. DPMJET-III modeli Gribov-Glauber formalizmi [34, 35, 36] ile bağlantılı olarak Çiftli Parton Modeline [37] dayanmaktadır. Bu olay üreticisinde parton model kullanılarak oluşturulan hadronların yüksek enerjilerde çarpışmaları analiz edilir ve Gribov-Glauber formalizmi ile çekirdek-çekirdek çarpışmaları nükleon etkileşimleri düzeyinde açıklanır [34, 35, 36]. Parçacıkların sicim parçalanma süreçleri ile oluştuğu DPMJET- III modeli, yumuşak ve sert saçılma olaylarını birleşik bir şekilde ele alır. Yumuşak olaylar Reggeon teorisine göre parametrelendirilirken, pertürbatif Kuantum Renk Dinamiği 
(KRD)sert olayları tanımlamak için kullanılır [27]. Modelde çoklu parton etkileşimleri için PHOJET olay oluşturucusu ve parton konfigürasyonlarının parçalanması için PYTHIA Lund modeli kullanılır [27].
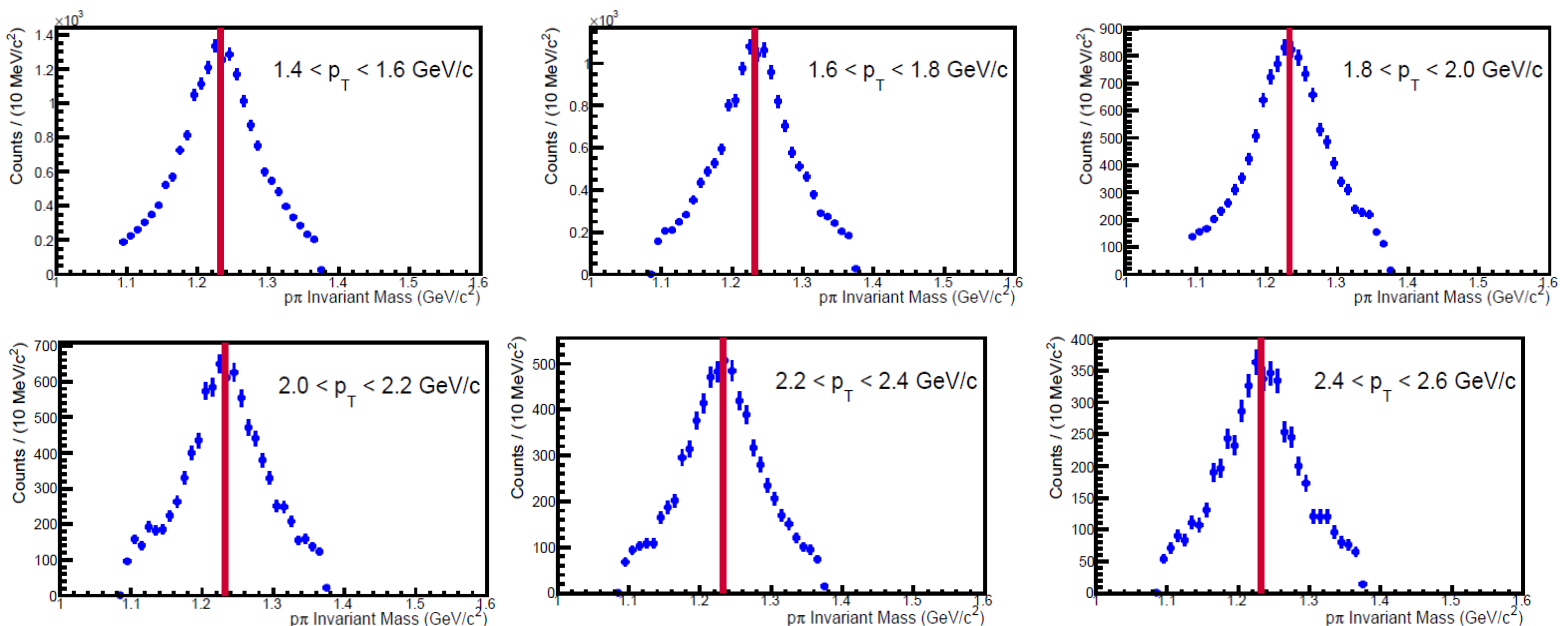

Şekil 6.DPMJET olay üretici ile elde edilmiş ${\sqrt{\mathrm{S}_{\mathrm{NN}}}}=5.02 \mathrm{TeV}$ enerjili p-Pb çarpışmaları sonucu oluşan $\Delta(1232)^{++}$parçacığının1.4 -2.6 p aralığı için değişmez kütle dağılımı. Şekilde kırmızı çizgiler parçacığın PDG tarafından kabul edilmiş kütle değerini temsil etmektedir.

DPMJET-III olay üreticisinde ${\sqrt{\mathrm{S}_{\mathrm{NN}}}}=5.02 \mathrm{TeV}$ enerjili proton kurşun $(\mathrm{p}-\mathrm{Pb}$ ) çarpışmaları sonucu oluşan $105.9 \times 10^{6}$ olay analiz edilmiştir. Bu olaylarda parçacık tanımlaması kullanılarak 1.4 ile $2.6 \mathrm{GeV} / \mathrm{c} \mathrm{p}_{\mathrm{T}}$ aralığında $66.3 \times 10^{3} \Delta(1232)^{++}$elde edilmiştir. Elde edilen rezonanslar 6 farklı enine momentum aralığında $(1.4-1.6,1.6$ - 1.8, 1.8 - 2.0, 2.0 - 2.2, 2.2 - 2.4 ve 2.4 - 2.6 GeV/c) incelenmiştir ve Şekil 6 'da bu aralıklarındaki dağılımları gösterilmiştir. Şekilde kırmızıçizgiler parçacığın PDG tarafından kabul edilmiş kütle değerini göstermektedir.

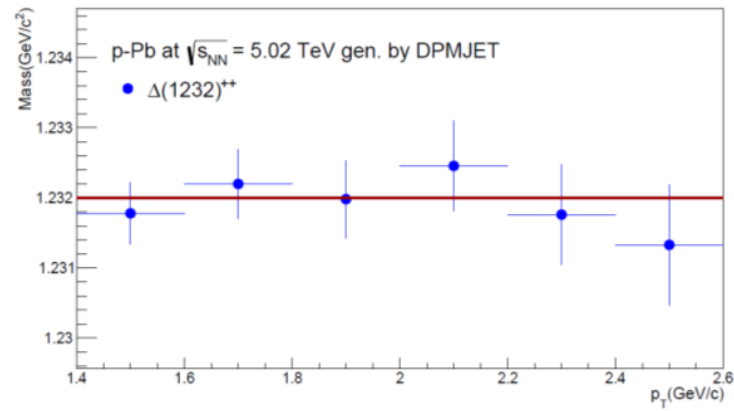

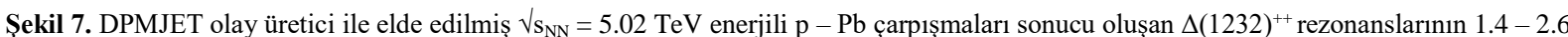
$\mathrm{p}_{\mathrm{T}}$ aralığı için elde edilmiş kütle değerleri. Grafikte kırmızı çizgi parçacı̆̆ın PDG tarafından kabul edilmiş kütle değerini ve hata çubukları istatiksel belirsizlikleri göstermektedir.

$\Delta(1232)^{++}$rezonansının kütlesi Şekil 6'da gösterilen dağılımlar kullanılarak 1.4 ile $2.6 \mathrm{GeV} / \mathrm{c}$ enine momentum aralığı için elde edilmiştir. Şekil 7'de parçacığın elde edilen kütlesinin enine momentuma bağlı değişimi yer almaktadır. Rölativistik çarpışma deneylerinde gözlemlenen rezonansın kütlesindeki enine momentuma bağlı kayma, spektral fonksiyonların hesaba katıldığı modeller kullanıldığında hesaplanabilmektedir [38]. Fakat PYTHIA ve PHOJET gibi sicim teorisine dayalı Monte Carlo olay üreticilerinde rölativistik spektral dalga fonksiyonları yerine sabit genlik değerine sahip Breit - Wigner fonksiyonları kullanılması nedeniyle kütlesel kaymaların benzetimi bu olay üreticileri ile yapılamamaktadır [39, 40]. Bu sebeple şekilde gösterildiği gibi DPMJET-III ile elde edilen $\Delta(1232)^{++}$kütle değerlerinde enine momentuma bağlı bir kayma gözlenmemiştir. 


\section{SONUÇLAR}

$\Delta(1232)^{++}$baryonik rezonansı rölativistik çarpışmalarda oluşan KGP fazı ile yaklaşık aynı yaşam süresine sahip protonun uyarılmış bir durumudur. Bulunduğu ortamın kütle ve rezonans genişliği gibi karakteristik özelliklerini etkilenmesi, $\Delta(1232)^{++}$oluşumları incelenerek çarpışmalarda oluşan sistem dinamiğinin anlaşılmasına olanak sağlar. STAR deneyinde ${\sqrt{\mathrm{S}_{\mathrm{NN}}}}=200 \mathrm{GeV}$ enerjili orta hızlılık $(|\mathrm{y}|<0.5)$ değerlerinde $\mathrm{d}+\mathrm{Au}$ ve pp çarpışmaları sonucu oluşan $\Delta(1232)^{++}$rezonanslarının 0.2 ile $1.6 \mathrm{GeV} / \mathrm{c}$ enine momentum aralığında kütle değerleri incelenmiştir. Ayrıca ALICE deneyinde $\sqrt{\mathrm{s}}=7 \mathrm{TeV}$ enerjili orta hızlılık $(|\mathrm{y}|<0.5)$ değerlerinde pp çarpışmaları sonucu oluşan $\Delta(1232)^{++}$ve $\bar{\Delta}(1232)^{--}$rezonanslarının 0.4 ile $2.0 \mathrm{GeV} / \mathrm{c}$ enine momentum aralığ için kütle değerleri incelenmiştir. Yapılan deneysel çalışmalar $\Delta(1232)^{++}$rezonansının çarpışma sisteminden bağımsız olarak kütle değerlerinde enine momentuma bağlı bir kayma olduğunu göstermiştir. Bu durum, rezonansların sahip oldukları kısa yarı ömür nedeniyle ve bulundukları ortamın yoğunluğu ve sıcaklığının etkisiyle kütle gibi karakteristik özelliklerinin değiştiği görüşü ile açılanabilir. $\Delta(1232)^{++}$rezonans oluşumları Monte Carlo olay üreticilerinden biri olan ve parçacık oluşumlarının sicim teorisine dayandığı DPMJET - III ile üretilmiş ${\sqrt{\mathrm{s}_{\mathrm{NN}}}}=$ $5.02 \mathrm{TeV}$ enerjili p- $\mathrm{Pb}$ çarpışmalarında incelenmiştir. Bu çalışmada parçacık tanımlaması yapılarak baryonik rezonansların değişmez kütle dağılımı 1.4 ile $2.6 \mathrm{GeV} / \mathrm{c}$ enine momentum aralığı için elde edilmiştir. Simülasyonda $105.9 \times 10^{6}$ olay analiz edilmesine rağmen bu olaylardan seçili enine momentum aralığında yaklaşık \%0.06'si kadarında $\Delta(1232)^{++}$olduğu gözlenmiştir. Bu sonuç simülasyonun bu rezonansları ölçülenden daha az ürettiğini göstermektedir. Dağılımlardan elde edilen değerler enine momentuma bağlı incelendiğinde rezonansin kütlesinde herhangi bir kayma gözlenmemiştir. Bu durum modelde sabit genlik değerine sahip Breit Wigner fonksiyonları kullanılması sebebiyle kütlesel kaymaların benzetiminin yapılamaması ile açıklanır. Ayrıca bu durum modellemede etkileşim kanallarının çok kısa yarı ömre sahip rezonans oluşumları için yetersiz olduğu görüşünü destekler.

\section{TEŞEKKÜR}

Bu çalışma 119F302 numaralı TÜBİTAK-1001 projesi ve 2019TAEK(CERN)A5.H1.F5-23 numaralı TAEK projesi ile desteklenmiştir.

\section{KAYNAKLAR}

[1] LHC Study Group (1995). The Large Hadron Collider Conceptual Design Report, CERN/AC 95-05.

[2] Bryant, P. \& Evans L. (2008). LHC Machine. Journal of Instrumentation, 3, S08001. doi: 10.1088/1748 0221/3/08/S08001.

[3] Harrison, M., Ludlam, T. \& Ozaki, S. (2003). RHIC Project over view. Nuclear Instruments and Methods in Physics Research A, 499, 235-244.

[4] Alice Collab. (2014). Performance of the ALICE Experiment at the CERN LHC. International Journal of Modern Physics A, 29, 1430044. doi:10.1142/S0217751X14300440

[5] Star Collab. (2003). STAR detector overview. Nuclear Instruments and Methods in Physics Research A, 499, 624-632. doi: 10.1016/S0168-9002(02)01960-5.

[6] Phenix Collab. (2003). PHENIX detector overview. Nuclear Instruments and Methods in Physics Research A, 499, 469-479.

[7] Tawfik, A. \& Shalaby, A. G. (2015). Balance Function in High-Energy Collisions. Advances in High Energy Physics, 186812.

[8] Markert, C. (2005). What do we learn from Resonance Production in Heavy Ion Collisions? Journal of Physics G: Nuclear and Particle Physics, 31 (4), 169-178.

[9] Torrieri, G. \& Rafelski, J. (2001). Strange Hadron Resonances as a Signature of Freeze-Out Dynamics. Physics Letters B, 509, 239-245.

[10] Bleicher, M. \& Aichelin, J. (2002). Strange resonance production: Probing chemical and thermal freeze-out in relativistic heavy ion collisions. Physics Letters B, 530(1-4), 81-87.

[11] Gyulassy, M. (2003). The QGP discovered at RHIC. Paper presented at the Proceedings of the NATO Advanced Study Institute: Structure and Dynamics of Elementary Matter, Kemer, Turkey.

[12] Phenix Collab. (2005). Formation of dense partonic matter in relativistic nucleus-nucleus collisions at RHIC: experimental evaluation by the PHENIX Collaboration. Nuclear Physics A, 757 (1-2), 184-283.

[13] Phobos Collab. (2005). The PHOBOS perspective on discoveries at RHIC. Nuclear Physics A, 757(1-2), 28101. 
[14] Star Collab. (2005). Experimental and theoretical challenges in the search for the quark-gluon plasma: the STAR Collaboration's critical assessment of the evidence from RHIC collisions. Nuclear Physics A, 757, 102-183.

[15] Brahms Collab. (2005). Quark-gluon plasma and color glass condensate at RHIC? The perspective from the Brahms experiment. Nuclear Physics A, 757 (1-2), 1-27.

[16] Particle Data Group Collab. (2018). Review of particle physics. Physics. Review D, 98, 030001.

[17] Bleicher, M. \& Aichelin, J. (2002). Strange resonance production: Probing chemical and thermal freeze-out in relativistic heavy ion collisions. Physics Letters B, 530(1-4), 81-87.

[18] Star Collab. (2008). Hadronic resonance production in $\mathrm{d}+\mathrm{Au}$ collisions at ${\sqrt{\mathrm{S}_{\mathrm{NN}}}}=200 \mathrm{GeV}$ at RHIC. Physics Review C, 78, 044906. doi:10.1103/PhysRevC.78.044906

[19] Particle Data Group Collab. (2014).Chinese Physics C, 38, 090001.

[20] Hote, D. (1994). About Resonance Signal Extraction From Multi particle Data: Combinatorics and Event Mixing Methods. Nuclear Instruments and Methods in Physics Research A, 337, 544-556.

[21] Star Collab. (2005). K*(892) Resonance Production in Au+Au and p+p Collisions at $\sqrt{s}=200 \mathrm{GeV}$ at $\mathrm{STAR}$. Physics Review C, 71, 064902.

[22] Rapp, R. (2003). Pi+ Pi- Emission in High-Energy Nuclear Collisions. Nuclear Physics A, 725, $254-268$.

[23] Shuryak, E. V. \& Brown, G. (2003).Matter-induced modification of resonances at RHIC freezeout. Nuclear Physics A, 717, 322-335.

[24] Star Collab. (2002). Coherent $\rho^{0}$ Production in Ultra peripheral Heavy-Ion Collisions. Physics Review Letter, 89, 272302.

[25] Kolb, P. F., \&Prakash, M. (2003). Spectroscopy of resonance decays in high-energy heavy-ion experiments. Physics Review C, 67,044902.

[26] Alice Collab. (2012). Study of short-lived resonances with the ALICE experiment at the LHC.Paper presented at the Proceedings of the 2nd International Conference on Particle Physics: In Memoriam Engin Arik and Her Colleagues, Istanbul, Turkey.

[27] Roesler, S., Engel, R. \& Ranft, J.(2000). The Monte Carlo event generator DPMJET-III. SLAC-PUB-8740, 1033-1038.

[28] Ranft, J. (1995). Dual parton model at cosmic ray energies. Physics Review D, 51 (1), 64-84.

[29] Ranft, J. (1999). DPMJET version II.5: Sampling of hadron-hadron, hadron-nucleus and nucleus-nucleus interactions at accelerator and cosmic ray energies according to the two-component dual parton model: Code manual, Preprint University of Siegen SI-99-6.

[30] Engel, R., Ranft, J. \& Roesler, S. (1997). Photo production off nuclei and point like photon interactions. I. Cross sections and nuclear shadowing. Physics Review D, 55, 6957.

[31] Roesler, S., Engel, R. \& Ranft, J. (1998). Photo production off nuclei: Particle and jet production. Physics Review D, 57, 2889.

[32] Engel, R. (1995). Photo production within the two component dual parton model. 1. Amplitudes and crosssections. Zeitschriftfür Physik C Particles and Fields, 66, 203-214.

[33] Engel, R. \& Ranft, J. (1996). Hadronic photon-photon interactions at high energies. Physics Review D, 54, 4244.

[34] Glauber, R. J. (1959). Lectures in Theoretical Physics. Interscience, New York, Volume 1, 315.

[35] Gribov, V. N. (1969). Glauber corrections and the interaction between high-energy hadrons and nuclei. Soviet Physics - The Journal of Experimental and Theoretical Physics, 29, 483.

[36] Gribov, V. N. (1970). Interaction of gamma quanta and electrons with nuclei at high-energies. Soviet Physics - The Journal of Experimental and Theoretical Physics, 30, 709.

[37] Capella, A., Sukhatme, U., Tan, C.I. \& Tran Thanh Van, J. (1994). Dual parton model. Physics Reports, 236 (4-5), 225-329.

[38] Hees, H. \&Rapp, R. (2018). Delta (1232) and Nucleon Spectral Functions in Hot Hadronic Matter. Physics Letter B, 606, 59-66.

[39] Weil, J. (2008). Dilepton Production in Elementary Nuclear Reactions within a BUU Transport Model. Diploma Tezi, Justus Liebig University, Teorik Fizik Enstitüsü, Giessen.

[40] Sjostrand, T., Mrenna S. \& Skands P. (2006). PYTHIA 6.4 Physics and Manual. Journal of High Energy Physics, 026. doi:10.1088/1126-6708/2006/05/026 